\title{
Quantization of the electromagnetic field outside static black holes and its application to low-energy phenomena
}

\author{
Luís C. B. Crispino \\ Instituto de Fisica Teórica, Universidade Estadual Paulista, Rua Pamplona 145, 01405-900, São Paulo, São Paulo, Brazil \\ and Departamento de Fisica, Universidade Federal do Pará, Campus Universitário do Guamá, 66075-900, Belém, Pará, Brazil \\ Atsushi Higuchi \\ Department of Mathematics, University of York, Heslington, York YO10 5DD, United Kingdom \\ George E. A. Matsas \\ Instituto de Fisica Teórica, Universidade Estadual Paulista, Rua Pamplona 145, 01405-900, São Paulo, São Paulo, Brazil
}

(Received 24 January 2001; published 11 May 2001)

\begin{abstract}
We discuss the Gupta-Bleuler quantization of the free electromagnetic field outside static black holes in the Boulware vacuum. We use a gauge which reduces to the Feynman gauge in Minkowski spacetime. We also discuss its relation with gauges used previously. Then we apply the low-energy sector of this field theory to investigate some low-energy phenomena. First, we discuss the response rate of a static charge outside the Schwarzschild black hole in four dimensions. Next, motivated by string physics, we compute the absorption cross sections of low-energy plane waves for the Schwarzschild and extreme Reissner-Nordström black holes in arbitrary dimensions higher than three.
\end{abstract}

DOI: 10.1103/PhysRevD.63.124008

PACS number(s): 04.70.Dy, 04.62.+v

\section{INTRODUCTION}

The prediction that black holes should thermally evaporate [1] has sparked much interest in quantum field theory in curved spacetimes. One of the difficulties in studying fields in Schwarzschild [2] and other black hole spacetimes, even when the fields are non-interacting, stems from the fact that the solutions to the field equations are functions whose properties are not well known. ${ }^{1}$ In the low-frequency regime, however, the situation is much simpler in Schwarzschild spacetime. In this regime, the mode functions of the massless scalar field are well known [4]. The present authors and Sudarsky used this fact to find the response rate of a static scalar source [5] and an analytic approximation for the emission rate of low-energy particles from classical sources [6] outside the Schwarzschild black hole in closed form. On the other hand, the field equations of the electromagnetic field in a black hole spacetime are not decoupled and are difficult to analyze in the Lorenz gauge. However, if we require the field to be divergence-free on a two-sphere (the spherical Coulomb gauge), the equations for the physical modes reduce to decoupled scalar field equations. Furthermore, solutions in terms of familiar special functions can be found in the lowenergy regime. These observations enabled us recently to calculate the response rate of a static electric charge outside a Schwarzschild black hole in closed form [7].

In this paper we examine free quantum electrodynamics in static spherically symmetric spacetimes of arbitrary dimensions in a modified Feynman gauge. (This gauge is closely related to the $A_{0}=0$ gauge used by Cognola and Lecca [8] and reduces to the Feynman gauge in Minkowski

\footnotetext{
${ }^{1}$ See Ref. [3] for some known properties in the Schwarzschild
} case. spacetime.) Then, we calculate some low energy quantities in electrodynamics outside spherically symmetric black holes. First, we review the calculation of the response rate of a static charge outside the four-dimensional Schwarzschild black hole in the Unruh vacuum [9]. Next we calculate the low energy absorption cross sections of photons for the Schwarzschild and extreme Reissner-Nordström black holes in arbitrary dimensions higher than three, extending some results obtained by Gubser [10] using a method [11,12] based on the Newman-Penrose formalism [13].

The paper is organized as follows. In Sec. II we present the mode functions of the electromagnetic field in the spacetime of a spherically symmetric black hole in our modified Feynman gauge. Then we discuss the corresponding quantum theory and show how the Gupta-Bleuler condition (see, e.g., [14]) is implemented to obtain the physical states. In Sec. III we compare the physical modes in the spherical Coulomb gauge [7] with the ones obtained in the modified Feynman gauge. In Sec. IV we review the calculation of the response rate of a static charge outside a four-dimensional Schwarzschild black hole. In Secs. V and VI we present the photon absorption cross sections by the Schwarzschild and extreme Reissner-Nordström black holes of arbitrary dimensions higher than three. In Sec. VII we summarize the main results and make some remarks. In Appendix A we compute some components of field-strength two-point function in Minkowski spacetime using spherical polar coordinates and show that they agree with those obtained using Cartesian coordinates. In Appendix B a summation formula for Legendre functions used in Sec. IV is derived. In Appendix C, a formula which relates the absorption probability to the absorption cross section is derived in arbitrary dimensions. We use the metric signature $(+--\ldots-)$ and the natural units with $G=\hbar=c=1$ throughout this paper. 


\section{GUPTA-BLEULER QUANTIZATION IN A MODIFIED FEYNMAN GAUGE}

In this section we analyze the field equations for the electromagnetic field in spherically symmetric and static spacetimes in a modified Feynman gauge. Then we discuss the Gupta-Bleuler quantization in this gauge.

The line element of the spacetime we study is

$$
d \tau^{2}=f(r) d t^{2}-h(r) d r^{2}-r^{2} d s_{p}^{2},
$$

where $d s_{p}^{2}$ is the line element of a unit $p$-sphere. We assume that $f(r)$ and $h(r)$ are positive for $r>r_{H}$ and that both $f(r)$ and $h(r)^{-1}$ have simple zeros or both have double zeros at $r=r_{H}$, where $r_{H}$ is the horizon radius. We also assume that $f(r), h(r)^{-1} \rightarrow 1$ as $r \rightarrow \infty$. (Most results in this section, however, are independent of these assumptions.) Let us introduce the Wheeler tortoise coordinate $r^{*}$ by

$$
\frac{d r^{*}}{d r}=\sqrt{\frac{h}{f}}
$$

Then $r^{*}(r)$ is a monotonic function with domain $\left(r_{H},+\infty\right)$ and range in $(-\infty,+\infty)$. Let us define for any two functions $q_{1}\left(r^{*}\right)$ and $q_{2}\left(r^{*}\right)$ the inner product

$$
\left\langle q_{1}, q_{2}\right\rangle=\int_{-\infty}^{+\infty} d r^{*} \overline{q_{1}\left(r^{*}\right)} q_{2}\left(r^{*}\right),
$$

where the overline denotes complex conjugation.

The Lagrangian density of the electromagnetic field in a modified Feynman gauge is

$$
\mathcal{L}_{\mathrm{F}}=\sqrt{-g}\left[-\frac{1}{4} F_{\mu \nu} F^{\mu \nu}-\frac{1}{2} G^{2}\right]
$$

with

$$
G=\nabla^{\mu} A_{\mu}+K^{\mu} A_{\mu},
$$

where the vector $K^{\mu}$ is independent of $A_{\mu}$. Hence the equations of motion are

$$
\nabla_{\nu} F^{\nu \mu}+\nabla^{\mu} G-K^{\mu} G=0 .
$$

Here we choose

$$
K^{\mu}=\left(0, f^{\prime} /(f h), 0,0\right),
$$

in which case Eq. (2.5) is written as

$$
G=\frac{1}{f} \partial_{t} A_{t}-\sqrt{\frac{f}{h}} \frac{1}{r^{p}} \partial_{r}\left[\frac{r^{p}}{\sqrt{f h}} A_{r}\right]-\frac{1}{r^{2}} \tilde{\nabla}^{i} A_{i} .
$$

Here $i$ denotes angular variables on the unit $p$-sphere $S^{p}$ with metric $\tilde{\eta}_{i j}$ and inverse metric $\tilde{\eta}^{i j}$ [with signature $(+\cdots+)], \tilde{\nabla}_{i}$ is the associated covariant derivative on $S^{p}$ and $\tilde{\nabla}^{i} \equiv \tilde{\eta}^{i j} \tilde{\nabla}_{j}$. This choice for $K^{\mu}$ is convenient because the equation for $A_{t}$ decouples from the other ones. The field equations (2.6) become

$$
\begin{gathered}
-\frac{1}{f} \partial_{t}^{2} A_{t}+\sqrt{\frac{f}{h}} \frac{1}{r^{p}} \partial_{r}\left[\frac{r^{p}}{\sqrt{f h}} \partial_{r} A_{t}\right]+\frac{1}{r^{2}} \tilde{\nabla}^{2} A_{t}=0 \\
-\frac{1}{f} \partial_{t}^{2} A_{r}+\frac{1}{f} \partial_{r}\left[\sqrt{\frac{f}{h}} \frac{f}{r^{p}} \partial_{r}\left(\frac{r^{p}}{\sqrt{f h}} A_{r}\right)\right]+\frac{1}{r^{2}} \tilde{\nabla}^{2} A_{r} \\
+\frac{1}{f} \partial_{r}\left(\frac{f}{r^{2}}\right) \tilde{\nabla}^{i} A_{i}=0, \\
-\frac{1}{f} \partial_{t}^{2} A_{i}+\frac{r^{2-p}}{\sqrt{f h}} \partial_{r}\left(\sqrt{\frac{f}{h}} r^{p-2} \partial_{r} A_{i}\right)-\frac{r^{2}}{f h} \partial_{r}\left(\frac{f}{r^{2}}\right) \partial_{i} A_{r} \\
+\frac{1}{r^{2}}\left[\tilde{\nabla}^{j}\left(\tilde{\nabla} A_{i}-\tilde{\nabla}_{i} A_{j}\right)+\partial_{i}\left(\tilde{\nabla}^{j} A_{j}\right)\right]=0,
\end{gathered}
$$

where $\tilde{\nabla}^{2} \equiv \tilde{\eta}_{i j} \tilde{\nabla}^{i} \widetilde{\nabla}^{j}$.

We shall now describe a complete set of solutions $A_{\mu}^{(\lambda n ; \omega l m)}$. We assign $\lambda$ the value 0 for what we call the non-physical modes, 1 or 2 for the physical modes and 3 for the pure-gauge modes. (These modes will be given below.) The label $n$ distinguishes between modes incoming from the past null infinity $\mathcal{J}^{-}$(denoted with $n=\leftarrow$ ) and those coming out from the past horizon $H^{-}$(denoted with $\left.n=\rightarrow\right){ }^{2}$ The solutions with $A_{\mu}^{(0 n ; \omega l m)}=0(\mu \neq t)$, and

$$
A_{t}^{(0 n ; \omega l m)}=R_{\omega l}^{(0 n)}(r) Y_{l m} e^{-i \omega t}
$$

will be called "non-physical modes" because they satisfy the field equations (2.9)-(2.11) but not the gauge condition $G=0$. Here $Y_{l m}$ is a scalar spherical harmonic on the unit $p$-sphere with $\tilde{\nabla}^{2} Y_{l m}=-l(l+p-1) Y_{l m}$, where $l$ $=0,1,2, \ldots$ and $m$ denotes a set of $p-1$ integers $\left(m_{1}, \ldots, m_{p-1}\right)$ satisfying $l \geqslant m_{p-1} \geqslant \ldots \geqslant m_{2} \geqslant\left|m_{1}\right|$. (See Ref. [15] for a concise description of spherical harmonics on the $p$-sphere.) They are normalized as

$$
\int d \Omega_{p} \overline{Y_{l m}} Y_{l^{\prime} m^{\prime}}=\delta_{l l^{\prime}} \delta_{m m^{\prime}}
$$

where $d \Omega_{p}$ is the volume element of the unit $p$-sphere. The function $R_{\omega l}^{(0 n)}(r)$ satisfies

$$
\left[\frac{\omega^{2}}{f}+\sqrt{\frac{f}{h}} \frac{1}{r^{p}} \frac{d}{d r}\left(\frac{r^{p}}{\sqrt{f h}} \frac{d}{d r}\right)-\frac{l(l+p-1)}{r^{2}}\right] R_{\omega l}^{(0 n)}=0 .
$$

We will determine the normalization of the functions $R_{\omega l}^{(0 n)}(r)$ later. The pure-gauge modes are given as

$$
A_{\mu}^{(3 n ; \omega l m)}=\nabla_{\mu} \Lambda^{(n \omega l m)}
$$

\footnotetext{
${ }^{2}$ Here we treat only the solutions proportional to $e^{-i \omega t}$ with $\omega$ $\neq 0$. Thus, if there is any nonzero static field, we will be considering fluctuation about that solution.
} 
where

$$
\Lambda^{(n \omega l m)}=\frac{i}{\omega} R_{\omega l}^{(0 n)}(r) Y_{l m} e^{-i \omega t}
$$

[As usual, the pure-gauge modes satisfy the field equations (2.9) $-(2.11)$ and $G=0$.$] The other independent solutions$ $(\lambda=1,2)$, which represent physical degrees of freedom, i.e., which satisfy the field equations and $G=0$ but are not pure gauge, will be chosen to have $A_{t}=0$. Those with $A_{r} \neq 0$ are given as

$$
A_{r}^{(1 n ; \omega l m)}=R_{\omega l}^{(1 n)}(r) Y_{l m} e^{-i \omega t}
$$

where

$$
\begin{aligned}
& {\left[\frac{\omega^{2}}{f}-\frac{l(l+p-1)}{r^{2}}\right] R_{\omega l}^{(1 n)}(r)} \\
& \quad+\frac{1}{r^{2}} \frac{d}{d r}\left[\sqrt{\frac{f}{h}} r^{2-p} \frac{d}{d r}\left(\frac{r^{p}}{\sqrt{f h}} R_{\omega l}^{(1 n)}(r)\right)\right]=0 .
\end{aligned}
$$

Note here that the condition $G=0$ cannot be solved if $l$ $=0$. Hence, we have $l \geqslant 1$. The corresponding angular components can be found by solving the condition $G=0$ as

$$
A_{i}^{(1 n ; \omega l m)}=\frac{r^{2-p}}{l(l+p-1)} \sqrt{\frac{f}{h}} \frac{d}{d r}\left[\frac{r^{p}}{\sqrt{f h}} R_{\omega l}^{(1 n)}\right] \partial_{i} Y_{l m} e^{-i \omega t} .
$$

We call these modes " physical modes I.', The other set of physical solutions can be obtained by letting $A_{t}=A_{r}=0$ and

$$
A_{i}^{(2 n ; \omega l m)}=R_{\omega l}^{(2 n)}(r) Y_{i}^{(l m)} e^{-i \omega t},
$$

where

$$
\begin{gathered}
{\left[\frac{\omega^{2}}{f}+\frac{1}{\sqrt{f h} r^{p-2}} \frac{d}{d r}\left(\sqrt{\frac{f}{h}} r^{p-2} \frac{d}{d r}\right)\right.} \\
\left.-\frac{(l+1)(l+p-2)}{r^{2}}\right] R_{\omega l}^{(2 n)}(r)=0 .
\end{gathered}
$$

Here, the $Y_{i}^{(l m)}$ are divergence-free vector spherical harmonics on the unit $p$-sphere satisfying

$$
\tilde{\nabla}^{k}\left(\tilde{\nabla}_{k} Y_{i}^{(l m)}-\tilde{\nabla}_{i} Y_{k}^{(l m)}\right)=-(l+1)(l+p-2) Y_{i}^{(l m)}
$$

and

$$
\int d \Omega_{p} \tilde{\eta}^{i j} \overline{Y_{i}^{(l m)}} Y_{j}^{\left(l^{\prime} m^{\prime}\right)}=\delta_{l l^{\prime}} \delta_{m m^{\prime}} .
$$

(See, e.g., Refs. [15,16].) We call these modes "physical modes II.' Physical modes I and II obtained here (and restricted to four dimensions) are identical with those in the $A_{0}=0$ gauge [8].
In order to discuss Gupta-Bleuler quantization of this field it is convenient to introduce a generalized Klein-Gordon product of classical solutions of Eq. (2.6). We first define

$$
\Pi^{\mu \nu} \equiv \frac{1}{\sqrt{-g}} \frac{\partial \mathcal{L}_{\mathrm{F}}}{\partial\left[\nabla_{\mu} A_{\nu}\right]}=-\left[F^{\mu \nu}+g^{\mu \nu} G\right],
$$

where $G$ is given in Eq. (2.5). Note that $\sqrt{-g} \Pi^{t \nu}$ is the canonical conjugate momentum of $A_{\nu}$. We write

$$
\left.\Pi^{(\zeta) \mu \nu} \equiv \Pi^{\mu \nu}\right|_{A_{\mu}=A_{\mu}^{(\zeta)}}
$$

for any solution $A_{\mu}^{(\zeta)} \equiv A_{\mu}^{(\lambda n ; \omega l m)}$. For any two (complex) solutions $A_{\mu}^{(\zeta)}$ and $A_{\mu}^{\left(\zeta^{\prime}\right)}$ we define

$$
W^{\mu}\left[A^{(\zeta)}, A^{\left(\zeta^{\prime}\right)}\right] \equiv i\left[\overline{A_{\nu}^{(\zeta)}} \Pi^{\left(\zeta^{\prime}\right) \mu \nu}-\overline{\Pi^{(\zeta) \mu \nu}} A_{\nu}^{\left(\zeta^{\prime}\right)}\right] .
$$

The field equations ensure that this current is conserved. As a result, the generalized Klein-Gordon inner product defined by

$$
\left(A^{(\zeta)}, A^{\left(\zeta^{\prime}\right)}\right) \equiv \int_{\Sigma} d \Sigma_{\mu} W^{\mu}\left[A^{(\zeta)}, A^{\left(\zeta^{\prime}\right)}\right]
$$

where $d \Sigma_{\mu} \equiv d \sigma n_{\mu}$, is independent of the Cauchy surface $\Sigma$ [17]. (Here $d \sigma$ is the volume element of the Cauchy surface $\Sigma$ with a normal unit vector $n^{\mu}$.) Note that $\left(A^{(\zeta)}, A^{\left(\zeta^{\prime}\right)}\right)$ $=\overline{\left(A^{\left(\zeta^{\prime}\right)}, A^{(\zeta)}\right)}$. This guarantees that the norm defined through Eq. (2.27) is real (and positive definite for the subset of physical solutions with positive frequency). By working explicitly with the definition (2.27), we find in general that on a $t=$ const surface

$$
\begin{aligned}
\left(A^{(\zeta)}, A^{\left(\zeta^{\prime}\right)}\right)= & -i \int d^{p+1} \mathbf{x} \sqrt{-g} f^{-1} g^{\mu \nu} \\
& \times\left(\overline{A_{\mu}^{(\zeta)}} \partial_{t} A_{\nu}^{\left(\zeta^{\prime}\right)}-A_{\nu}^{\left(\zeta^{\prime}\right)} \partial_{t} \overline{A_{\mu}^{(\zeta)}}\right) .
\end{aligned}
$$

It is important to note that pure-gauge modes are orthogonal to any mode satisfying $G=0$ and, as a result, $\nabla_{\nu} F^{\nu \mu}$ $=0$. This can be shown as follows. Suppose that $A_{\mu}^{(\zeta)}$ and $A_{\mu}^{\left(\zeta^{\prime}\right)}$ satisfy the condition $G=0$. Then since $\Pi^{(\zeta) \mu \nu}=$

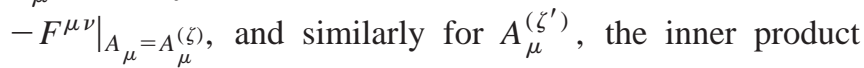
(2.27) can be written as $\left(A^{(\zeta)}, A^{\left(\zeta^{\prime}\right)}\right)=\left(A^{(\zeta)}, A^{\left(\zeta^{\prime}\right)}\right)_{\text {inv }}$ where

$$
\left(A^{(\zeta)}, A^{\left(\zeta^{\prime}\right)}\right)_{\mathrm{inv}} \equiv i \int_{\Sigma} d \Sigma_{\mu}\left[\overline{A_{\nu}^{(\zeta)}} F^{\left(\zeta^{\prime}\right) \nu \mu}-\overline{F^{(\zeta) \nu \mu}} A_{\nu}^{\left(\zeta^{\prime}\right)}\right]
$$

Now, let $A_{\mu}^{(\zeta)}=\nabla_{\mu} \Lambda^{(\zeta)}$ be a pure-gauge mode. Then

$$
\begin{aligned}
\left(A^{(\zeta)}, A^{\left(\zeta^{\prime}\right)}\right)_{\mathrm{inv}} & =i \int_{\Sigma} d \Sigma_{\mu} F^{\left(\zeta^{\prime}\right) \nu \mu} \nabla_{\nu} \overline{\Lambda^{(\zeta)}} \\
& \left.=i \int_{\Sigma} d \Sigma_{\mu} \nabla_{\nu} \overline{\left(\overline{\Lambda^{(\zeta)}}\right.} F^{\left(\zeta^{\prime}\right) \nu \mu}\right) \\
& =0
\end{aligned}
$$


since $F^{\left(\zeta^{\prime}\right) \nu \mu}$ is anti-symmetric. ${ }^{3}$ Thus $\left(A^{(\zeta)}, A^{\left(\zeta^{\prime}\right)}\right)=0$ if $A_{\mu}^{(\zeta)}$ is a pure-gauge mode and $A^{\left(\zeta^{\prime}\right)}$ is a physical or pure-gauge mode, i.e.,

$$
\left(A^{(3 n ; \omega l m)}, A^{\left(3 n^{\prime} ; \omega^{\prime} l^{\prime} m^{\prime}\right)}\right)=0
$$

$\left(A^{(3 n ; \omega l m)}, A^{\left(1 n^{\prime} ; \omega^{\prime} l^{\prime} m^{\prime}\right)}\right)=0, \quad\left(A^{(3 n ; \omega l m)}, A^{\left(2 n^{\prime} ; \omega^{\prime} l^{\prime} m^{\prime}\right)}\right)=0$.

Next let us examine the inner product of the non-physical solutions. By letting

$$
R_{\omega l}^{(0 n)}(r) \equiv \sqrt{f} r^{-p / 2} \varphi_{\omega l}^{(0 n)}(r),
$$

we find from Eq. (2.14)

$$
\left(\omega^{2}+\frac{d^{2}}{d r^{* 2}}-V_{0}\left(r^{*}\right)\right) \varphi_{\omega l}^{(0 n)}=0
$$

where $r^{*}$ is given in Eq. (2.2) and

$$
\begin{aligned}
V_{0}\left[r^{*}(r)\right]= & f \frac{l(l+p-1)}{r^{2}}-\frac{f}{h}\left[\frac{p(2-p)}{4 r^{2}}-\frac{f^{\prime 2}}{2 f^{2}}\right. \\
& \left.+\frac{p}{4 r}\left(\frac{f^{\prime}}{f}+\frac{h^{\prime}}{h}\right)+\frac{f^{\prime \prime}}{2 f}-\frac{f^{\prime} h^{\prime}}{4 f h}\right] .
\end{aligned}
$$

For spacetimes where $f(r)$ and $h(r)^{-1}$ have simple zeros at $r=r_{H}$, we find $V_{0}\left[r^{*}\left(r_{H}\right)\right] \neq 0$ in general. If $V_{0}\left[r^{*}\left(r_{H}\right)\right]$ $>0$, then the non-physical modes coming out from the horizon have frequencies satisfying $\omega^{2}>V_{0}\left[r^{*}\left(r_{H}\right)\right]$. In particular, for Reissner-Nordström spacetime in $(p+2)$ dimensions [18], where

$$
f(r)=h(r)^{-1}=\left[1-\left(\frac{r_{+}}{r}\right)^{p-1}\right]\left[1-\left(\frac{r_{-}}{r}\right)^{p-1}\right]
$$

with $r_{ \pm}^{p-1}=M \pm \sqrt{M^{2}-Q^{2}}$ (so that $r_{H}=r_{+}$), we find

$$
V_{0}\left[r^{*}\left(r_{H}\right)\right]=\frac{(p-1)^{2}}{\left(2 r_{+}\right)^{2}}\left[1-\left(\frac{r_{-}}{r_{+}}\right)^{p-1}\right]^{2} \text {. }
$$

As expected, we have $\left\langle\varphi_{\omega l}^{(0 \rightarrow)}, \varphi_{\omega^{\prime} l}^{(0 \leftarrow)}\right\rangle=0$, i.e., the solutions of Eq. (2.34) incoming from $H^{-}$and those incoming from $\mathcal{J}^{-}$are orthogonal to one another with respect to the inner product defined by Eq. (2.3). By normalizing these solutions so that

$$
\begin{aligned}
& \varphi_{\omega l}^{(0 \rightarrow)} \approx \sqrt{\omega / \tilde{\omega}}\left(e^{i \tilde{\omega} r^{*}}+\mathcal{R}_{\omega l}^{(0 \rightarrow)} e^{-i \tilde{\omega} r^{*}}\right) \quad\left(r^{*} \rightarrow-\infty\right), \\
& \varphi_{\omega l}^{(0 \leftarrow)} \approx e^{-i \omega r^{*}}+\mathcal{R}_{\omega l}^{(0 \leftarrow)} e^{i \omega r^{*}} \quad\left(r^{*} \rightarrow+\infty\right),
\end{aligned}
$$

${ }^{3}$ Note that Eq. (2.30) would hold even if $\Lambda^{(\zeta)}$ was an arbitrary function. This shows that $\left(A^{(\zeta)}, A^{\left(\zeta^{\prime}\right)}\right)_{\text {inv }}$ is gauge invariant. up to a phase factor, where $\tilde{\omega}^{2}=\omega^{2}-V_{0}\left[r^{*}\left(r_{H}\right)\right] \geqslant 0$ and $\mathcal{R}_{\omega l}^{(0 \rightarrow)}$ and $\mathcal{R}_{\omega l}^{(0 \leftarrow)}$ are constants (with $\left|\mathcal{R}_{\omega l}^{(0 \rightarrow)}\right|=\left|\mathcal{R}_{\omega l}^{(0 \leftarrow)}\right|$ ), we have

$$
\left\langle\varphi_{\omega l}^{(0 \leftarrow)}, \varphi_{\omega^{\prime} l}^{(0 \leftarrow)}\right\rangle=\left\langle\varphi_{\omega l}^{(0 \rightarrow)}, \varphi_{\omega^{\prime} l}^{(0 \rightarrow)}\right\rangle=2 \pi \delta\left(\omega-\omega^{\prime}\right) .
$$

Now, by using Eq. (2.28) we find

$$
\begin{aligned}
\left(A^{(0 n ; \omega l m)}, A^{\left(0 n^{\prime} ; \omega^{\prime} l^{\prime} m^{\prime}\right)}\right) & =-2 \omega\left\langle\varphi_{\omega l}^{(0 n)}, \varphi_{\omega^{\prime} l^{\prime}}^{\left(0 n^{\prime}\right)}\right\rangle \delta_{n n^{\prime}} \delta_{l l^{\prime}} \delta_{m m^{\prime}} \\
& =-4 \pi \omega \delta_{n n^{\prime}} \delta_{l l^{\prime}} \delta_{m m^{\prime}} \delta\left(\omega-\omega^{\prime}\right)
\end{aligned}
$$

where we have used $\delta\left(\tilde{\omega}-\tilde{\omega}^{\prime}\right)=(\tilde{\omega} / \omega) \delta\left(\omega-\omega^{\prime}\right)$. Noting that $A^{(0 n ; \omega l m)}$ and $A^{(3 n ; \omega l m)}$ have the same $t$-components, we immediately obtain from Eqs. (2.28) and (2.31) that

$$
\left(A^{(0 n ; \omega l m)}, A^{\left(3 n^{\prime} ; \omega^{\prime} l^{\prime} m^{\prime}\right)}\right)=-4 \pi \omega \delta_{n n^{\prime}} \delta_{l l^{\prime}} \delta_{m m^{\prime}} \delta\left(\omega-\omega^{\prime}\right) .
$$

Next let us examine the physical modes. By letting

$$
R_{\omega l}^{(1 n)}(r) \equiv \frac{\sqrt{l(l+p-1)}}{\omega}(f h)^{1 / 2} r^{-p / 2-1} \varphi_{\omega l}^{(1 n)}(r),
$$

we find from Eq. (2.18)

$$
\left(\omega^{2}+\frac{d^{2}}{d r^{* 2}}-V_{1}\left(r^{*}\right)\right) \varphi_{\omega l}^{(1 n)}=0
$$

where

$$
\begin{aligned}
V_{1}\left[r^{*}(r)\right]= & f \frac{l(l+p-1)}{r^{2}}+\frac{p(p-2)}{4 r^{2}} \frac{f}{h} \\
& -\frac{(p-2)}{4} \frac{f}{h r}\left(\frac{f^{\prime}}{f}-\frac{h^{\prime}}{h}\right) .
\end{aligned}
$$

(We note that $V_{1} \rightarrow 0$ as $r \rightarrow r_{+}$for Reissner-Nordström spacetime unlike $V_{0}$.) We first note that the modes $A^{(1 n ; \omega l m)}$ are orthogonal to both the non-physical and pure-gauge modes. Next we note

$$
\left(A^{(1 n ; \omega l m)}, A^{\left(1 n^{\prime} ; \omega^{\prime} l^{\prime} m^{\prime}\right)}\right)=2 \omega\left\langle\varphi_{\omega l}^{(1 n)}, \varphi_{\omega^{\prime} l^{\prime}}^{\left(1 n^{\prime}\right)}\right\rangle \delta_{n n^{\prime}} \delta_{l l^{\prime}} \delta_{m m^{\prime}} .
$$

Thus, by normalizing $\varphi_{\omega l}^{(1 n)}(r)$ as

$$
\begin{aligned}
& \varphi_{\omega l}^{(1 \rightarrow)} \approx e^{i \omega r^{*}}+\mathcal{R}_{\omega l}^{(1 \rightarrow)} e^{-i \omega r^{*}} \quad\left(r^{*} \rightarrow-\infty\right), \\
& \varphi_{\omega l}^{(1 \leftarrow)} \approx e^{-i \omega r^{*}}+\mathcal{R}_{\omega l}^{(1 \leftarrow)} e^{i \omega r^{*}} \quad\left(r^{*} \rightarrow+\infty\right),
\end{aligned}
$$

we have

$$
\left(A^{(1 n ; \omega l m)}, A^{\left(1 n^{\prime} ; \omega^{\prime} l^{\prime} m^{\prime}\right)}\right)=4 \pi \omega \delta_{n n^{\prime}} \delta_{l l^{\prime}} \delta_{m m^{\prime}} \delta\left(\omega-\omega^{\prime}\right)
$$


Finally, we let

$$
R_{\omega l}^{(2 n)}(r) \equiv r^{-(p-2) / 2} \varphi_{\omega l}^{(2 n)}(r) .
$$

Then from Eq. (2.21), we have

$$
\left(\omega^{2}+\frac{d^{2}}{d r^{* 2}}-V_{2}\left(r^{*}\right)\right) \varphi_{\omega l}^{(2 n)}=0,
$$

where

$$
\begin{aligned}
V_{2}\left[r^{*}(r)\right]= & f \frac{(l+1)(l+p-2)}{r^{2}}+\frac{(p-2)(p-4)}{4 r^{2}} \frac{f}{h} \\
& +\frac{(p-2)}{4} \frac{f}{h r}\left(\frac{f^{\prime}}{f}-\frac{h^{\prime}}{h}\right) .
\end{aligned}
$$

(We again note that $V_{2} \rightarrow 0$ as $r \rightarrow r_{+}$in Reissner-Nordström spacetime.) Notice that $V_{1}=V_{2}$ if $p=2$. Hence, in the fourdimensional case $\varphi_{\omega l}^{(1 n)}(r)$ and $\varphi_{\omega l}^{(2 n)}(r)$ satisfy the same equation. These modes can easily be shown to be orthogonal to the modes previously discussed. By normalizing the functions $\varphi_{\omega l}^{(2 n)}(r)$ in the same way as $\varphi_{\omega l}^{(1 n)}(r)$ [see Eqs. (2.44),(2.45)], we have

$$
\left(A^{(2 n ; \omega l m)}, A^{\left(2 n^{\prime} ; \omega^{\prime} l^{\prime} m^{\prime}\right)}\right)=4 \pi \omega \delta_{n n^{\prime}} \delta_{l l^{\prime}} \delta_{m m^{\prime}} \delta\left(\omega-\omega^{\prime}\right) .
$$

In order to quantize the field $A_{\mu}$, we impose the equaltime commutation relations on the field $\hat{A}_{\mu}$ and momentum $\hat{\Pi}^{t \mu}$ operators:

$$
\begin{gathered}
{\left[\hat{A}_{\mu}(t, \mathbf{x}), \hat{A}_{\nu}\left(t, \mathbf{x}^{\prime}\right)\right]=\left[\hat{\Pi}^{t \mu}(t, \mathbf{x}), \hat{\Pi}^{t \nu}\left(t, \mathbf{x}^{\prime}\right)\right]=0,} \\
{\left[\hat{A}_{\mu}(t, \mathbf{x}), \hat{\Pi}^{t \nu}\left(t, \mathbf{x}^{\prime}\right)\right]=\frac{i \delta_{\mu}^{\nu}}{\sqrt{-g}} \delta^{p+1}\left(\mathbf{x}-\mathbf{x}^{\prime}\right),}
\end{gathered}
$$

where $\mathbf{x}$ and $\mathbf{x}^{\prime}$ represent all spatial coordinates. The field $\hat{A}_{\mu}$ can be expanded using the modes we have obtained before:

$$
\hat{A}_{\mu}(t, \mathbf{x})=\sum_{\rho} \int_{-\infty}^{+\infty} \frac{d \omega}{\sqrt{4 \pi|\omega|}} A_{\mu}^{(\omega \rho)}(t, \mathbf{x}) a_{\omega \rho}
$$

where $A_{\mu}^{(\omega \rho)}$ is proportional to $e^{-i \omega t}, A_{\mu}^{(-\omega \rho)} \equiv \overline{A_{\mu}^{(\omega \rho)}}, a_{-\omega \rho}$ $\equiv a_{\omega \rho}^{\dagger}$ and $\rho$ labels discrete quantum numbers. The commutation relations $(2.50),(2.51)$ are equivalent to the following commutation relations in the "symplectically smeared", form as is the case for scalar fields [19]:

$$
\left[\left(A^{(\zeta)}, \hat{A}\right),\left(\hat{A}, A^{\left(\zeta^{\prime}\right)}\right)\right]=\left(A^{(\zeta)}, A^{\left(\zeta^{\prime}\right)}\right) .
$$

Since the inner product must be $t$ independent, the inner product of $A_{\mu}^{(\omega \rho)}$ and $A_{\mu}^{\left(\omega^{\prime} \rho^{\prime}\right)}$ can be nonzero only if $\omega$ $=\omega^{\prime}$. Thus, we can write

$$
\left(A^{(\omega \rho)}, A^{\left(\omega^{\prime} \rho^{\prime}\right)}\right)=M^{\rho \rho^{\prime}} \delta\left(\omega-\omega^{\prime}\right) .
$$

By using Eq. (2.54) in Eq. (2.53) one finds

$$
M^{\rho_{1} \rho_{2}}\left[a_{\omega \rho_{2}}, a_{\omega^{\prime} \rho_{3}}^{\dagger}\right] M^{\rho_{3} \rho_{4}}=4 \pi \omega M^{\rho_{1} \rho_{4}} \delta\left(\omega-\omega^{\prime}\right) .
$$

Then, since $M^{\rho \rho^{\prime}}$ is invertible in our case, we find

$$
\left[a_{\omega \rho}, a_{\omega^{\prime} \rho^{\prime}}^{\dagger}\right]=4 \pi \omega\left(M^{-1}\right)_{\rho \rho^{\prime}} \delta\left(\omega-\omega^{\prime}\right) .
$$

Note here that we immediately have $\left[a_{\omega \rho}, a_{\omega^{\prime} \rho^{\prime}}\right]=0$ for $\omega, \omega^{\prime}>0$ by letting $\omega^{\prime} \rightarrow-\omega^{\prime}$ in Eq. (2.56). By using the Klein-Gordon inner products computed above, we find the following commutators:

$$
\begin{aligned}
{\left[a_{\omega l m}^{(3 n)}, a_{\omega^{\prime} l^{\prime} m^{\prime}}^{\left(3 n^{\prime}\right) \dagger}\right] } & =-\left[a_{\omega l m}^{(0 n)}, a_{\omega^{\prime} l^{\prime} m^{\prime}}^{\left(3 n^{\prime}\right) \dagger}\right] \\
& =\delta_{n n^{\prime}} \delta_{l l^{\prime}} \delta_{m m^{\prime}} \delta\left(\omega-\omega^{\prime}\right), \\
{\left[a_{\omega l m}^{(1 n)}, a_{\omega^{\prime} l^{\prime} m^{\prime}}^{\left(1 n^{\prime}\right) \dagger}\right] } & =\left[a_{\omega l m}^{(2 n)}, a_{\omega^{\prime} l^{\prime} m^{\prime}}^{\left(2 n^{\prime}\right) \dagger}\right] \\
& =\delta_{n n^{\prime}} \delta_{l l^{\prime}} \delta_{m m^{\prime}} \delta\left(\omega-\omega^{\prime}\right)
\end{aligned}
$$

with all other commutators vanishing. The Gupta-Bleuler condition [14] requires that any physical state $\mid$ phys $\rangle$ satisfy

$$
\left.\hat{G}^{(+)} \mid \text {phys }\right\rangle=0,
$$

where $\hat{G}^{(+)}$is the positive-frequency part of $\hat{G}=\nabla^{\mu} \hat{A}_{\mu}$ $+K^{\mu} \hat{A}_{\mu}$. Since this quantity is nonvanishing only for $A_{\mu}^{(0 n ; \omega l m)}$, this condition is equivalent to

$$
\left.a_{\omega l m}^{(0 n)} \mid \text { phys }\right\rangle=0 \quad \text { for all }(n, \omega, l, m) \quad(\text { with } \omega>0) .
$$

(We let $\omega>0$ below.) The Boulware vacuum $|0\rangle$ [20] is defined by requiring that it be annihilated by all $a_{\omega l m}^{(\lambda n)}$ operators $(\lambda=0,1,2,3)$. Note that the states obtained by applying any number of creation operators excluding $a_{\omega l m}^{(3 n) \dagger}$ are all physical states. Any state of the form $a_{\omega l m}^{(3 n) \dagger} \mid$ phys $\rangle$ is unphysical because

$$
\left.\left.a_{\omega^{\prime} l^{\prime} m^{\prime}}^{\left(0 n^{\prime}\right)} a_{\omega l m}^{(3 n) \dagger} \mid \text { phys }\right\rangle=-\delta_{n n^{\prime}} \delta_{l l^{\prime}} \delta_{m m^{\prime}} \delta\left(\omega-\omega^{\prime}\right) \mid \text { phys }\right\rangle \neq 0 .
$$

Note also that the physical states of the form $a_{\omega l m}^{(0 n) \dagger} \mid$ phys $\rangle$ have zero norm and are orthogonal to any physical states. Thus, as is well known, a physical state $\mid$ phys $\left._{1}\right\rangle$ can be regarded as equivalent to any state of the form $\left|\mathrm{phys}_{1}\right\rangle$ $+a_{\omega l m}^{(0 n) \dagger} \mid$ phys $\left._{2}\right\rangle$. We can take as the representative elements the states obtained by applying $a_{\omega l m}^{(\lambda n) \dagger}, \lambda=1,2$, on $|0\rangle$.

Unphysical particles created by $a_{\omega l m}^{(3 n) \dagger}$ will be in thermal equilibrium in the Hartle-Hawking vacuum [21] for a static black hole if we require the gauge-fixed two-point function be non-singular on the horizons as in the scalar case [22]. There will also be a flux of unphysical particles in the Unruh vacuum. Therefore, technically speaking, the HartleHawking and Unruh vacua are unphysical if we impose the Gupta-Bleuler condition using the positive-frequency notion in the Boulware vacuum. Fortunately, the Becchi-RouetStora-Tyutin (BRST) quantization does not suffer from this 
problem since the physical state condition does not refer to any notion of positive frequency [23]. This issue, however, will not concern us in the following, since all our calculations will be at the tree level.

Mode expansions in spherical polar coordinates are not very widely used and are quite different from the ordinary method using Cartesian coordinates. For this reason, we compute in Appendix A some components of field-strength two-point function in four-dimensional Minkowski spacetime using spherical polar coordinates. We find that they agree with the standard results obtained in Cartesian coordinates, as they should.

\section{THE PHYSICAL MODES IN THE SPHERICAL COULOMB GAUGE}

As we have seen, the modified Feynman gauge defined by Eq. (2.7) is useful in finding explicit mode functions. We also noted that this gauge results in the same physical modes as in the $A_{0}=0$ gauge. There is another convenient gauge for finding physical modes, i.e., the spherical Coulomb gauge, $\tilde{\nabla}^{i} A_{i}=0$. (One can readily show that any vector $A_{\mu}$ is gaugeequivalent to a vector satisfying this gauge condition.) Let us discuss the relation of the physical modes obtained in this gauge and those found in the previous section. ${ }^{4}$

It is clear that the physical modes $A_{\mu}^{(2 n ; \omega l m)}$ in the previous section are also in the spherical Coulomb gauge. The other independent physical modes must have $A_{i}=0$ in this gauge. First we note that if $A_{t}$ and $A_{r}$ are assumed to be spherically symmetric, then we find from the equations $\nabla^{\mu} F_{\mu t}=\nabla^{\mu} F_{\mu r}=0$ that $F_{t r}$ is $t$-independent and $\left(r^{p} / \sqrt{f h}\right) F_{t r}$ is $r$-independent. Hence, if in addition the modes are assumed to be proportional to $e^{-i \omega t}$ with $\omega \neq 0$, they must be pure gauge because $F_{t r}=0$. (A timeindependent solution has $F_{t r} \propto \sqrt{f h} / r^{p}$. This will represent a static Coulomb field, which we will disregard here.) To find the solutions which are not spherically symmetric we let $A_{t}, A_{r} \propto Y_{l m} e^{-i \omega t}$. From the equation $\nabla^{\mu} F_{\mu i}=0$ we find

$$
-i \omega A_{t}=\sqrt{\frac{f}{h}} \frac{1}{r^{p-2}} \partial_{r}\left[\sqrt{\frac{f}{h}} r^{p-2} A_{r}\right] .
$$

By substituting this and $A_{i}=0$ in $\nabla^{\mu} F_{\mu r}=0$, we find that $A_{r}^{\left(1^{\prime} n ; \omega l m\right)}=R_{\omega l}^{\left(1^{\prime} n\right)}(r) Y_{l m} e^{-i \omega t}$ (here we use primed indices to refer to the spherical Coulomb gauge), where

$$
\begin{aligned}
& {\left[\frac{\omega^{2}}{f}-\frac{l(l+p-1)}{r^{2}}\right] R_{\omega l}^{\left(1^{\prime} n\right)}(r)} \\
& \quad+\frac{1}{f} \frac{d}{d r}\left[\sqrt{\frac{f}{h}} \frac{1}{r^{p-2}} \frac{d}{d r}\left(\sqrt{\frac{f}{h}} r^{p-2} R_{\omega l}^{\left(1^{\prime} n\right)}(r)\right)\right]=0 .
\end{aligned}
$$

\footnotetext{
${ }^{4}$ Again, we only treat solutions proportional to $e^{-i \omega t}$ with $\omega \neq 0$.
}

The component $A_{t}^{\left(1^{\prime} n ; \omega l m\right)}$ can be found from Eq. (3.1). (Then, one can readily show that $\nabla^{\mu} F_{\mu t}=0$.) Indeed one can verify that these modes are related to the ones in the modified Feynman gauge by a gauge transformation:

$$
A_{\mu}^{(1 n ; \omega l m)} \rightarrow A_{\mu}^{\left(1^{\prime} n ; \omega l m\right)}=A_{\mu}^{(1 n ; \omega l m)}-\nabla_{\mu} \Phi^{(1 n ; \omega l m)},
$$

where

$$
\Phi^{(1 n ; \omega l m)}=\frac{r^{2-p}}{l(l+p-1)} \sqrt{\frac{f}{h}} \frac{d}{d r}\left(\frac{r^{p}}{\sqrt{f h}} R_{\omega l}^{(1 n)}\right) Y_{l m} e^{-i \omega t}
$$

This gives us

$$
\begin{aligned}
& A_{t}^{\left(1^{\prime} n ; \omega l m\right)}=\frac{i \omega r^{2-p}}{l(l+p-1)} \sqrt{\frac{f}{h}} \frac{d}{d r}\left(\frac{r^{p}}{\sqrt{f h}} R_{\omega l}^{(1 n)}\right) Y_{l m} e^{-i \omega t} \\
& A_{r}^{\left(1^{\prime} n ; \omega l m\right)}=\frac{\omega^{2} r^{2}}{l(l+p-1)} \frac{1}{f} R_{\omega l}^{(1 n)} Y_{l m} e^{-i \omega t}
\end{aligned}
$$

\section{RESPONSE RATE OF A STATIC CHARGE OUTSIDE A FOUR-DIMENSIONAL SCHWARZSCHILD BLACK HOLE}

Here we briefly review, in the context of the previous two sections, the calculation of response rate in the Unruh vacuum [9] of a static electric charge in Schwarzschild spacetime performed in Ref. [7].

The line element of the four-dimensional Schwarzschild spacetime is

$$
d \tau^{2}=f(r) d t^{2}-f(r)^{-1} d r^{2}-r^{2} d s_{2}^{2}
$$

with $f(r)=1-2 M / r$, where $d s_{2}^{2}=d \theta^{2}+\sin ^{2} \theta d \phi^{2}$ is the line element of the unit two-sphere. (The horizon radius is $r_{H}$ $=2 M$.) We compute the response rate of the static electric charge to the Hawking radiation present in the Unruh vacuum. The charge is placed at $(r, \theta, \phi)=\left(r_{0}, \theta_{0}, \phi_{0}\right)$ as described by the current density

$$
j^{\mu}=\delta_{t}^{\mu} \frac{q}{r^{2} \sin \theta} \delta\left(r-r_{0}\right) \delta\left(\theta-\theta_{0}\right) \delta\left(\phi-\phi_{0}\right)
$$

Since this current density is static, it couples to photons with zero energy. It turns out that the rate of response of this current to a single photon vanishes. However, the BoseEinstein distribution of the thermal photons coming out of the horizon diverges at zero energy. This makes the rates of absorption and stimulated emission indefinite. This ambiguity can be resolved by replacing the current density (4.2) by $j^{\mu}=\left(j^{t}, j^{r}, 0,0\right)$, where

$$
j^{t}=\frac{\sqrt{2} q \cos E t}{r^{2} \sin \theta} \delta\left(r-r_{0}\right) \delta\left(\theta-\theta_{0}\right) \delta\left(\phi-\phi_{0}\right),
$$




$$
j^{r}=\frac{\sqrt{2} q E \sin E t}{r^{2} \sin \theta} \Theta\left(r-r_{0}\right) \delta\left(\theta-\theta_{0}\right) \delta\left(\phi-\phi_{0}\right) .
$$

Here, $\Theta(x)=1$ if $x>0$ and $\Theta(x)=0$ if $x \leqslant 0$. (The factor of $\sqrt{2}$ is necessary to make the time average of the squared charge equal to $q^{2}$.) We take the limit $E \rightarrow 0$ in the end, assuming that the rate is continuous at $E=0$. This continuity has been verified in Minkowski spacetime [24].

Since the current (4.2) is conserved, it does not interact with pure-gauge particles corresponding to the creation operators $a_{\omega l m}^{(3 n) \dagger}$. It does interact with the states created by $a_{\omega l m}^{(0 n) \dagger}$ but these have zero norm and do not contribute to physical probabilities. Hence, as usual, we need to consider only the physical modes. Note also that only physical modes I are relevant because $A_{t}=A_{r}=0$ for physical modes II.

Now, the transition amplitude from the Boulware vacuum $|0\rangle$ to the state $|1 n ; \omega l m\rangle=a_{\omega l m}^{(1 n) \dagger}|0\rangle$ by the classical current density $j^{\mu}$ is given by

$$
\mathcal{A}_{\omega l m}^{n}=i \int d^{4} x \sqrt{-g} j^{\mu}\left\langle 1 n ; \omega \operatorname{lm}\left|\hat{A}_{\mu}\right| 0\right\rangle .
$$

Let us recast Eq. (4.5) as

$$
\mathcal{A}_{\omega l m}^{n} \equiv 2 \pi i \mathcal{T}_{\omega l m}^{n} \delta(\omega-E),
$$

where $\mathcal{T}_{\omega l m}^{n}$ will be calculated later. The corresponding transition rate, i.e. transition probability per asymptotic proper time, is expressed as

$$
\mathcal{R}_{\omega l m}^{n}=2 \pi\left|\mathcal{T}_{\omega l m}^{n}\right|^{2} \delta(\omega-E) .
$$

Now we take into account that in the Unruh vacuum there is a thermal flux of photons coming out of the horizon with inverse temperature $\beta=8 \pi M$ (with no particles coming from infinity). In the limit $E \rightarrow 0$, only the absorption and stimulated emission of these thermal photons contribute to the response rate. For this reason, we treat only the modes coming out of the horizon $(n=\rightarrow)$. The total response rate due to these modes with fixed angular momentum, in which absorption, spontaneous and stimulated emissions are all taken into account, is

$$
R_{E l m}=\int_{0}^{+\infty} d \omega \mathcal{R}_{\omega l m}^{\rightarrow}\left(\frac{2}{e^{\beta \omega}-1}+1\right)=2 \pi \operatorname{coth} \frac{\beta E}{2}\left|\mathcal{T}_{E l m}\right|^{2}
$$

Thus, in the limit $E \rightarrow 0$ we find

$$
R_{0 l m}=\lim _{E \rightarrow 0}\left|\mathcal{T}_{E l m}\right|^{2} /(2 M E) .
$$

Next $\mathcal{T}_{E l m}$ will be calculated. The equation satisfied by the function $q_{\omega l}(r) \equiv(2 M / r) \varphi_{\omega l}^{(1 \rightarrow)}(r)$ can be obtained from Eq. (2.41) with $p=2$ as

$$
\begin{aligned}
& \frac{d}{d z}\left[\left(1-z^{2}\right) \frac{d q_{\omega l}}{d z}\right]+\left[l(l+1)-\frac{2}{z+1}-M^{2} \omega^{2} \frac{(z+1)^{3}}{z-1}\right] q_{\omega l} \\
& \quad=0
\end{aligned}
$$

where $z \equiv r / M-1$. For small $\omega$, the wave is almost completely reflected back to the horizon. This implies that

$$
\begin{array}{r}
\varphi_{\omega l}^{(1 \rightarrow)} \approx-2 \sin \left[\omega\left(r^{*}-\tilde{r}_{\omega}\right)\right] \approx-2 \omega r^{*}+\text { const } \\
\left(r-r_{H} \ll \omega^{2} r_{H}^{3},\left|\omega r^{*}\right| \ll 1\right),
\end{array}
$$

where $\tilde{r}_{\omega}$ are constants. The minus sign has been inserted for later convenience. The Wheeler tortoise coordinate $r^{*}$ defined by Eq. (2.2) is written in Schwarzschild spacetime as

$$
r^{*}=r+2 M \ln \left(\frac{r}{2 M}-1\right)
$$

Thus, Eq. (4.11) can be written as

$$
\varphi_{\omega l}^{(1 \rightarrow)} \approx-4 M \omega \ln (z-1) \quad\left(r-r_{H} \ll \omega^{2} r_{H}^{3},\left|\omega r^{*}\right| \ll 1\right) .
$$

Now, Eq. (4.10) can be solved explicitly for $\omega=0$. By choosing the solution for $q_{\omega l}$ such that $\varphi_{\omega l}^{(1 \rightarrow)}$ $=[r /(2 M)] q_{\omega l} \rightarrow$ tends to zero as $z \rightarrow+\infty$, we find in the small $\omega$ limit

$$
\varphi_{\omega l}^{(1 \rightarrow)}=4 M \omega(z+1)\left[Q_{l}(z)-\frac{(z-1)}{l(l+1)} \frac{d Q_{l}(z)}{d z}\right],
$$

where this has been normalized such that Eqs. (4.13) and (4.14) are in agreement at $r \approx r_{H}$.

From gauge invariance of the amplitude (4.5) it is clear that we may use the modes in the spherical Coulomb gauge, $A_{\mu}^{\left(1^{\prime} \rightarrow ; \omega l m\right)}$, in place of those in the modified Feynman gauge $A_{\mu}^{(1 \rightarrow ; \omega l m)}$. Then the contribution from the $r$-component will be suppressed in the low energy limit due to extra factors of $\omega$ [see Eq. (3.6)]. Therefore we need to consider only the $t$-component in this limit. The $t$-component can be found in the small $\omega$ limit from Eqs. (2.40), (3.5) and (4.14) as

$$
A_{t}^{\left(1^{\prime} \rightarrow ; \omega l m\right)}=\frac{4 i \omega(z-1)}{\sqrt{l(l+1)}} \frac{d Q_{l}(z)}{d z} Y_{l m}(\theta, \phi) e^{-i \omega t},
$$

where we have used the Legendre equation satisfied by $Q_{l}(z)$. By using Eq. (4.15) in Eq. (4.5) and comparing the result with Eq. (4.6), we obtain

$$
\mathcal{T}_{\omega l m}=-i \frac{\sqrt{2 \omega} q\left(z_{0}-1\right)}{\sqrt{\pi l(l+1)}} \frac{d Q_{l}\left(z_{0}\right)}{d z_{0}} \bar{Y}_{l m}\left(\theta_{0}, \phi_{0}\right)
$$

where $z_{0}=r_{0} / M-1$. Then we find the proper response rate of the charge as [see Eq. (4.9)] 


$$
\frac{R_{0 l m}}{\sqrt{f\left(r_{0}\right)}}=\frac{q^{2}\left(z_{0}-1\right)^{2}}{\pi M l(l+1) \sqrt{f\left(r_{0}\right)}}\left[\frac{d Q_{l}\left(z_{0}\right)}{d z_{0}}\right]^{2}\left|Y_{l m}\left(\theta_{0}, \phi_{0}\right)\right|^{2},
$$

where the factor $\sqrt{f\left(r_{0}\right)}$ appears because here we are calculating the transition rate per proper time of the charge. We can sum over $l$ and $m$ by using

$$
\sum_{m=-l}^{l}\left|Y_{l m}\left(\theta_{0}, \phi_{0}\right)\right|^{2}=(2 l+1) /(4 \pi)
$$

and

$$
\sum_{l=1}^{\infty} \frac{2 l+1}{l(l+1)}\left[\frac{d Q_{l}(z)}{d z}\right]^{2}=\frac{2 Q_{1}(z)}{\left(z^{2}-1\right)^{2}} .
$$

A derivation of this formula is given in Appendix B. Thus, the total transition probability per proper time of the charge is

$$
R^{\mathrm{tot}}=\sum_{l=1}^{+\infty} \sum_{m=-l}^{l} \frac{R_{0 l m}}{\sqrt{f\left(r_{0}\right)}}=\frac{q^{2} a\left(r_{0}\right)}{2 \pi^{2}} Q_{1}\left(r_{0} / M-1\right),
$$

where $a\left(r_{0}\right)=M r_{0}^{-2} / \sqrt{f\left(r_{0}\right)}$ is the proper acceleration of the charge.

It is of course possible to work with the modes in the modified Feynman gauge directly and obtain the same result. If we do so, the contribution will come from the $r$-component of the current because the $t$-components of physical modes I vanish.

\section{LOW ENERGY ABSORPTION CROSS SECTION OF THE SCHWARZSCHILD BLACK HOLE}

Here we consider the Schwarzschild black hole in $p+2$ dimensions given by the line element (2.1) with $f(r)=1$ $-\left(r_{H} / r\right)^{p-1}$. If the absorption probability of the black hole associated with physical modes I and II are $\mathcal{P}_{l}^{(1)}$ and $\mathcal{P}_{l}^{(2)}$, respectively, then the absorption cross section is given by

$$
\sigma=\frac{(2 \pi)^{p}}{p \Omega_{p} \omega^{p}} \sum_{l=1}^{\infty}\left[M_{l}^{(1)} \mathcal{P}_{l}^{(1)}+M_{l}^{(2)} \mathcal{P}_{l}^{(2)}\right],
$$

where $l$ and $\omega$ are the angular momentum and frequency, respectively, of the modes and $\Omega_{p}=2 \pi^{(p+1) / 2} / \Gamma[(p+1) / 2]$ is the volume of the unit $p$-sphere. Here, $M_{l}^{(1)}$ and $M_{l}^{(2)}$ are the multiplicities of the scalar and divergence-free vector spherical harmonics, respectively, on the $p$-sphere. These multiplicities are given by (see, e.g., Refs. $[15,16]$ )

$$
M_{l}^{(1)}=\frac{(2 l+p-1)(l+p-2) !}{(p-1) ! l !},
$$

and

$$
M_{l}^{(2)}=\frac{(2 l+p-1)(l+p-1) !}{(l+1)(l+p-2)(p-2) !(l-1) !} .
$$

Equation (5.1) is derived in Appendix C.

For either physical modes I or II, we have for large $r$ $\left(\approx r^{*}\right)$ that [see Eqs. $(2.41)$ and $\left.(2.47)\right]$

$$
\left\{\omega^{2}+\frac{d^{2}}{d r^{2}}-\left[l(l+p-1)+\frac{p(p-2)}{4}\right] \frac{1}{r^{2}}\right\} \varphi_{\omega l}^{(\lambda \leftarrow)}(r) \approx 0 .
$$

The asymptotic solution with the correct normalization, i.e. with the coefficient of $e^{-i \omega r}$ being one, is

$$
\varphi_{\omega l}^{(\lambda \leftarrow)}(r) \approx \sqrt{2 \pi \omega r} J_{l+(p-1) / 2}(\omega r) \quad\left(r_{H} \ll r\right) .
$$

Thus, for small $\omega r$

$$
\varphi_{\omega l}^{(\lambda \leftarrow)}(r) \approx C_{l}\left(r / r_{H}\right)^{l+p / 2} \quad\left(r_{H} \ll r \ll \omega^{-1}\right),
$$

where

$$
C_{l}=\frac{\sqrt{4 \pi}}{\Gamma(l+(p+1) / 2)}\left(\frac{\omega r_{H}}{2}\right)^{l+p / 2} .
$$

Now, near the horizon, where $V_{\lambda} \ll \omega^{2}(\lambda=1,2)$, these solutions behave like [see Eqs. (2.41) and (2.47)]

$$
\varphi_{\omega l}^{(\lambda \leftarrow)}(r) \approx D_{l}^{(\lambda)} e^{-i \omega r^{*}} \quad\left(r-r_{H} \ll \omega^{2} r_{H}^{3}\right),
$$

where the constants $D_{l}^{(\lambda)}$ will be determined later. Hence, for $\left|\omega r^{*}\right| \ll 1$ we have in this region $\varphi_{\omega l}^{(\lambda \leftarrow)}(r) \approx D_{l}^{(\lambda)}$ and the absorption probability is given by $\mathcal{P}_{l}^{(\lambda)}=\left|D_{l}^{(\lambda)}\right|^{2}$.

In order to find the coefficient $D_{l}^{(\lambda)}$, we solve the equations for $\varphi_{\omega l}^{(\lambda \leftarrow)}(r)$ with $\omega=0$. For physical modes I, it turns out that the function $r^{p} R_{\omega l}^{(1 \leftarrow)}$ satisfies a hypergeometric equation with the variable $w=1-\left(r / r_{H}\right)^{p-1}$. Thus, we find in the small $\omega$ limit

$$
\begin{aligned}
\varphi_{\omega l}^{(1 \leftarrow)}(r)= & D_{l}^{(1)}\left(r / r_{H}\right)^{1-p / 2} F(-(l+p-1) /(p-1), \\
& l /(p-1) ; 1 ; w) .
\end{aligned}
$$

This function approaches $D_{l}^{(1)}$ for $r \rightarrow r_{H}$ as required. By using the formula [25]

$$
F(\alpha, \beta ; \gamma ; w) \approx \frac{\Gamma(\gamma) \Gamma(\beta-\alpha)}{\Gamma(\beta) \Gamma(\gamma-\alpha)}(-w)^{-\alpha}
$$

$$
(\alpha<\beta,-w \gg 1)
$$

in Eq. (5.9), we find asymptotically

$$
\varphi_{\omega l}^{(1 \leftarrow)}(r) \approx D_{l}^{(1)} E_{l}^{(1)}\left(r / r_{H}\right)^{l+p / 2} \quad\left(r_{H} \ll r \ll \omega^{-1}\right),
$$

where

$$
E_{l}^{(1)}=\frac{\Gamma[(2 l+p-1) /(p-1)]}{\Gamma[l /(p-1)] \Gamma[(l+2 p-2) /(p-1)]} .
$$

By comparing Eqs. (5.6) and (5.11), we obtain

$$
\mathcal{P}_{l}^{(1)}=\left[C_{l} / E_{l}^{(1)}\right]^{2} .
$$


Thus, the absorption probability behaves like $\omega^{2 l+p}$.

Similarly, for physical modes II, we find in the small $\omega$ limit

$$
\begin{aligned}
\varphi_{\omega l}^{(2 \leftarrow)}(r)= & D_{l}^{(2)}\left(r / r_{H}\right)^{(p / 2-1)} F(-(l+1) /(p-1), \\
& (l+p-2) /(p-1) ; 1 ; w) .
\end{aligned}
$$

Then by using Eq. (5.10) we find asymptotically

$$
\varphi_{\omega l}^{(2 \leftarrow)}(r) \approx D_{l}^{(2)} E_{l}^{(2)}\left(r / r_{H}\right)^{l+p / 2} \quad\left(r_{H} \ll r \ll \omega^{-1}\right),
$$

where

$$
E_{l}^{(2)}=\frac{\Gamma[(2 l+p-1) /(p-1)]}{\Gamma[(l+p-2) /(p-1)] \Gamma[(l+p) /(p-1)]} .
$$

By comparing Eqs. (5.6) and (5.15), we obtain

$$
\mathcal{P}_{l}^{(2)}=\left[C_{l} / E_{l}^{(2)}\right]^{2} .
$$

Again, the absorption probability behaves like $\omega^{2 l+p}$. Therefore the physical modes with $l=1$ give the dominant contribution to the absorption probability, as expected.

By substituting $\mathcal{P}_{1}^{(1)}$ and $\mathcal{P}_{1}^{(2)}$ in Eq. (5.1), we obtain the total absorption cross section for low energy as

$$
\sigma=\frac{\omega^{2} r_{H}^{2} A_{H}}{2(p+1)}\left[\frac{2 p[\Gamma(p /(p-1))]^{4}}{[\Gamma((p+1) /(p-1))]^{2}}+1\right],
$$

where $A_{H}=\Omega_{p} r_{H}^{p}$ is the area of the black hole.

One would need to solve the differential equations satisfied by $\varphi_{\omega l}^{(\lambda \leftarrow)}$ numerically to find the low-energy absorption cross section for non-extreme Reissner-Nordström black holes. ${ }^{5}$ However, it is possible to calculate it analytically for the extreme Reissner-Nordström black hole. We turn to this problem in the next section.

\section{LOW ENERGY ABSORPTION CROSS SECTION OF THE EXTREME REISSNER-NORDSTRÖM BLACK HOLE}

The line element of the extreme Reissner-Nordström spacetime is given by Eq. (2.1) with $f(r)=h(r)^{-1}=[1$ $\left.-\left(r_{H} / r\right)^{p-1}\right]^{2}$. As in the Schwarzschild case, we have $\varphi_{\omega l}^{(\lambda \leftarrow)}(r) \approx C_{l}\left(r / r_{H}\right)^{l+p / 2}$ for $r_{H} \ll r \ll \omega^{-1}$ where $C_{l}$ is given by Eq. (5.7).

Now, let us analyze the physical modes close to the horizon. Concerning mode I, we write

$$
\left[\omega^{2}+\frac{d^{2}}{d r^{* 2}}-\frac{l(l+p-1)}{(p-1)^{2} r^{* 2}}\right] \varphi_{\omega l}^{(1 n)} \approx 0 \quad\left(-\omega^{2} r^{* 3} \gg r_{H}\right),
$$

${ }^{5}$ It is possible to obtain a result in a closed form if one takes the limit $\omega \rightarrow 0$ and $r_{+} \rightarrow r_{-}$simultaneously [11]. where we have used that the Wheeler tortoise coordinate $r^{*}$ [see Eq. (2.2)] behaves in this region as

$$
r^{*} \approx-\frac{(p-1)^{-2} r_{H}^{2}}{r-r_{H}}
$$

Hence we obtain

$$
\varphi_{\omega l}^{(1 \leftarrow)} \approx D_{l}^{(1)} \sqrt{\frac{-\pi \omega r^{*}}{2}} H_{\nu}^{(1)}\left(-\omega r^{*}\right) \quad\left(-\omega^{2} r^{* 3} \gg r_{H}\right)
$$

with

$$
\nu=\left[\frac{1}{4}+\frac{l(l+p-1)}{(p-1)^{2}}\right]^{1 / 2}=\frac{1}{2}+\frac{l}{p-1} .
$$

This solution behaves like $D_{l}^{(1)} e^{-i \omega r^{*}}$ (up to a phase factor) very close to the horizon $\left(-\omega r^{*} \gg 1\right)$, where the effective potential $V_{1}$ becomes negligible, as expected. Hence, the absorption probability is $\mathcal{P}_{l}^{(1)}=\left|D_{l}^{(1)}\right|^{2}$. Note that, for small $z$ with positive $\nu$, one has

$$
H_{\nu}^{(1)}(z) \approx-\frac{i \Gamma(\nu)}{\pi}\left(\frac{2}{z}\right)^{\nu}
$$

Hence,

$$
\begin{aligned}
\varphi_{\omega l}^{(1 \leftarrow)} \approx D_{l}^{(1)} K_{l}^{(1)}[ & {\left[\frac{(p-1)\left(r-r_{H}\right)}{r_{H}}\right]^{\nu-1 / 2} } \\
& \left(\left(\omega r_{H}\right)^{1 / 3} \ll-\omega r^{*} \ll 1\right),
\end{aligned}
$$

where

$$
K_{l}^{(1)}=-\frac{i \Gamma(\nu)}{\sqrt{\pi}}\left[\frac{2(p-1)}{\omega r_{H}}\right]^{\nu-1 / 2} .
$$

Again, in order to determine $D_{l}^{(1)}$, we solve the equation for $\varphi_{\omega l}^{(1 \leftarrow)}(r)$ in the small $\omega$ limit:

$$
\begin{aligned}
\varphi_{\omega l}^{(1 \leftarrow)}(r)= & D_{l}^{(1)} K_{l}^{(1)}\left(\frac{r}{r_{H}}\right)^{1-p / 2} \\
& \times\left(1-\frac{l}{l+p-1} w\right)(-w)^{l /(p-1)},
\end{aligned}
$$

where $w=1-\left(r / r_{H}\right)^{p-1}$, and we note that Eq. (6.8) has been normalized such that it agrees with Eq. (6.6) near the horizon. On the other hand, we find asymptotically

$$
\varphi_{\omega l}^{(1 \leftarrow)} \approx \frac{l}{l+p-1} D_{l}^{(1)} K_{l}^{(1)}\left(\frac{r}{r_{H}}\right)^{l+p / 2} \quad\left(r_{H} \ll r \ll \omega^{-1}\right) .
$$

By comparing this with Eq. (5.6) we find 


$$
\mathcal{P}_{l}^{(1)}=\left|\frac{(l+p-1) C_{l}}{l K_{l}^{(1)}}\right|^{2} .
$$

We note that $\mathcal{P}_{l}^{(1)} \propto \omega^{p+2 l p /(p-1)}$. Hence the dominant contribution to the scattering cross section comes from the $l$ $=1$ term:

$$
\begin{aligned}
\mathcal{P}_{1}^{(1)}= & \frac{4 \pi^{2} p^{2}}{\left[(p-1)^{1 /(p-1)} \Gamma[(p+3) / 2] \Gamma[(p+1) /(2 p-2)]\right]^{2}} \\
& \times\left(\frac{\omega r_{H}}{2}\right)^{2+p+2 /(p-1)}
\end{aligned}
$$

The contribution from physical modes II can be found in a similar manner. By substituting Eq. (6.2) in Eq. (2.47), we have near the horizon

$$
\left[\omega^{2}+\frac{d^{2}}{d r^{* 2}}-\frac{(l+1)(l+p-2)}{(p-1)^{2} r^{* 2}}\right] \varphi_{\omega l}^{(2 n)} \approx 0 \quad\left(-\omega^{2} r^{* 3} \gg r_{H}\right) .
$$

Then $\varphi_{\omega l}^{(2 \leftarrow)}$ will have the same form as $\varphi_{\omega l}^{(1 \leftarrow)}$ in Eq. (6.3) except that $\nu$ in Eq. (6.4) is replaced by

$$
\nu^{\prime}=\left[\frac{1}{4}+\frac{(l+1)(l+p-2)}{(p-1)^{2}}\right]^{1 / 2} .
$$

For $p \geqslant 3$, the contribution from physical mode II is suppressed compared to that from physical modes I since $\nu^{\prime}$ $>\nu$. For $p=2$, i.e. in four dimensions, Eqs. (6.1) and (6.12) satisfied by $\varphi_{\omega l}^{(1 n)}$ and $\varphi_{\omega l}^{(2 n)}$ are the same. Thus, the absorption probabilities of these two types of modes are equal for each $l$.

Hence, we obtain the following results:

$$
\begin{gathered}
\sigma=\frac{4}{3}\left(\omega r_{H}\right)^{4} A_{H} \quad \text { for } p=2, \\
\sigma=\frac{\pi p\left(\omega r_{H}\right)^{2+2 /(p-1)} A_{H}}{(p+1)[2(p-1)]^{2 /(p-1)}[\Gamma[(p+1) /(2 p-2)]]^{2}} \\
\text { for } p \geqslant 3 .
\end{gathered}
$$

Equation (6.13) is in agreement with the result derived by Gubser [10] . It is interesting to note that the low energy absorption cross section is proportional to a fractional power of $\omega$ if $p \geqslant 4$.

\section{CONCLUSIONS}

In this paper we showed that the field equations for free electrodynamics can be reduced to decoupled scalar field equations in a modified Feynman gauge in the spacetime of spherically symmetric black hole. (We also noted that the equations for the physical modes simplify in the spherical Coulomb gauge.) Then we examined the Gupta-Bleuler quantization in this modified Feynman gauge.

Next we reviewed the calculation of the response rate of a static charge outside the four-dimensional Schwarzschild black hole. It is easy to extend our result to the Schwarzschild black hole in arbitrary dimensions, though we cannot simplify the resulting infinite series. A closed-form expression for the response rate of a static source has been obtained for massless scalar field in four-dimensional ReissnerNordström black hole [26]. The extension of the results in this paper to the Reissner-Nordström black hole will also be possible in principle, but the result will probably not be expressible (even) as an infinite series of familiar special functions in any dimensions.

Finally we calculated the absorption cross sections of low energy photons by the Schwarzschild and extreme ReissnerNordström black holes in arbitrary dimensions. The corresponding cross section of massless scalar particles is known to be equal to the horizon area [27] as long as the black hole is spherically symmetric. No such universality holds for photons as our results and previous results [10] show. It is interesting that there are two modes, modes I and II, which behave differently in higher dimensions. It is also intriguing that the absorption cross section scales as a fractional power of the energy $\omega$, i.e. as $\omega^{2+p+2 /(p-1)}$ in $p+2$ dimensions. Finally, it would be interesting to compare the results here or similar results obtained using our gauge with those in string theory. (See, e.g., Ref. [10] and references therein for examples of such comparison.)

\section{ACKNOWLEDGMENTS}

L.C. and G.M. would like to acknowledge partial financial support from CAPES through the PICDT program and Conselho Nacional de Desenvolvimento Científico e Tecnológico, respectively.

\section{APPENDIX A: FIELD-STRENGTH TWO-POINT FUNCTION IN SPHERICAL POLAR COORDINATES IN FOUR-DIMENSIONAL MINKOWSKI SPACETIME}

Minkowski spacetime is the simplest spherically symmetric spacetime. In this appendix we compute some components of the two-point function

$$
G_{\mu \nu \rho \sigma}\left(x, x^{\prime}\right) \equiv\left\langle 0\left|\hat{F}_{\mu \nu}(x) \hat{F}_{\rho \sigma}\left(x^{\prime}\right)\right| 0\right\rangle
$$

in spherical polar coordinates and verify that they agree with those previously computed in Cartesian coordinates in fourdimensional Minkowski spacetime. We need to consider only physical modes because pure-gauge modes do not contribute to $\hat{F}_{\mu \nu}$ and the coefficient operators of the nonphysical modes have zero commutators with all operators appearing on the right-hand side of Eq. (A1).

Since there is no horizon, all modes come in from infinity and go out to infinity. Therefore, we do not need here the label $n=\leftarrow, \rightarrow$ which distinguished between the modes coming out of the horizon and those incoming from infinity. By the remark after Eq. (2.43) we have $\varphi_{\omega l}^{(1)}(r)$ $=2 \omega r j_{l}(\omega r)$, where $j_{l}(x)$ is the spherical Bessel function of order $l$, in four-dimensional Minkowski spacetime. Hence for physical modes I we have exactly 


$$
A_{r}^{(1 ; \omega l m)}=\frac{2 \sqrt{l(l+1)}}{r} j_{l}(\omega r) Y_{l m}(\theta, \phi) e^{-i \omega t},
$$

where $Y_{l m}(\theta, \phi)$ are the familiar scalar spherical harmonics on $S^{2}$. The $t r$-component of the corresponding field strength is

$$
F_{t r}^{(1 ; \omega l m)}=-i \frac{2 \sqrt{l(l+1)} \omega}{r} j_{l}(\omega r) Y_{l m}(\theta, \phi) e^{-i \omega t},
$$

since the $t$-component vanishes. These modes are sufficient for computing $G_{t r t r}\left(x, x^{\prime}\right)$ because physical modes II have $A_{t}=A_{r}=0$. We obtain with $x=(t, r, \theta, \phi)$ and $x^{\prime}$ $=\left(t^{\prime}, r^{\prime}, \theta^{\prime}, \phi^{\prime}\right)$

$$
\begin{aligned}
G_{t r t r}\left(x, x^{\prime}\right)= & \frac{1}{\pi r r^{\prime}} \sum_{l=1}^{\infty} l(l+1) \sum_{m=-l}^{l} Y_{l m}(\theta, \phi) \bar{Y}_{l m}\left(\theta^{\prime}, \phi^{\prime}\right) \\
& \times \int_{0}^{\infty} d \omega \omega j_{l}(\omega r) j_{l}\left(\omega r^{\prime}\right) e^{-i \omega\left(t-t^{\prime}\right)} \\
= & \frac{1}{8 \pi^{2}\left(r r^{\prime}\right)^{2}} \sum_{l=1}^{\infty} l(l+1)(2 l+1) \\
& \times P_{l}(\cos \gamma) Q_{l}\left[\frac{-\left(t-t^{\prime}-i \epsilon\right)^{2}+r^{2}+r^{\prime 2}}{2 r r^{\prime}}\right],
\end{aligned}
$$

where we have used in the last step the formulas

$$
\sum_{m=-l}^{l} Y_{l m}(\theta, \phi) \bar{Y}_{l m}\left(\theta^{\prime}, \phi^{\prime}\right)=\frac{2 l+1}{4 \pi} P_{l}(\cos \gamma),
$$

with $\cos \gamma=\cos \theta \cos \theta^{\prime}+\sin \theta \sin \theta^{\prime} \cos \left(\phi-\phi^{\prime}\right)$, and [25]

$$
\begin{aligned}
& \int_{0}^{\infty} d \omega \omega j_{l}(\omega r) j_{l}\left(\omega r^{\prime}\right) e^{-i \omega\left(t-t^{\prime}\right)} \\
& \quad=\frac{1}{2 r r^{\prime}} Q_{l}\left[\frac{-\left(t-t^{\prime}-i \epsilon\right)^{2}+r^{2}+r^{\prime 2}}{2 r r^{\prime}}\right] .
\end{aligned}
$$

We will simplify Eq. (A4) later.

Next we consider the component

$$
G_{\theta \phi \theta \phi}\left(x, x^{\prime}\right)=\frac{\sin \theta \sin \theta^{\prime}}{4} \widetilde{\epsilon}^{i j} \widetilde{\epsilon}^{i^{\prime} j^{\prime}} G_{i j i^{\prime} j^{\prime}}\left(x, x^{\prime}\right),
$$

where the anti-symmetric tensor on $S^{2}, \tilde{\epsilon}^{i j}$, has the component $\tilde{\epsilon}^{\theta \phi}=(\sin \theta)^{-1}$. (This definition differs from that of Regge and Wheeler [28] by a minus sign.) Only the modes $A_{\mu}^{(2 ; \omega l m)}$ contribute here. First, we have $\varphi_{\omega l}^{(2)}(r)$ $=2 \omega r j_{l}(\omega r)$. Then since $R_{\omega l}^{(2)}(r)=\varphi_{\omega l}^{(2)}(r)$ for $p=2$, we find

$$
A_{i}^{(2 ; \omega l m)}=2 \omega r j_{l}(\omega r) Y_{i}^{(l m)}(\theta, \phi) e^{-i \omega t},
$$

where the transverse vector spherical harmonics $Y_{i}^{(l m)}$ are given by

$$
Y_{i}^{(l m)}=\frac{\widetilde{\epsilon}_{i j} \tilde{\nabla}^{j} Y_{l m}}{\sqrt{l(l+1)}} .
$$

The corresponding field strength is given by

$$
\frac{1}{2} \widetilde{\epsilon}^{i j} F_{i j}^{(2 ; \omega l m)}=2 \sqrt{l(l+1)} \omega r j_{l}(\omega r) Y_{l m}(\theta, \phi) e^{-i \omega t} .
$$

Then in exactly the same way as for $G_{t r t r}$, we find

$$
\begin{aligned}
G_{\theta \phi \theta \phi}\left(x, x^{\prime}\right)= & \frac{\sin \theta \sin \theta^{\prime}}{8 \pi^{2}} \sum_{l=1}^{\infty} l(l+1)(2 l+1) \\
& \times P_{l}(\cos \gamma) Q_{l}\left[\frac{-\left(t-t^{\prime}-i \epsilon\right)^{2}+r^{2}+r^{\prime 2}}{2 r r^{\prime}}\right] .
\end{aligned}
$$

On the other hand one can calculate $G_{\alpha \beta \gamma \delta}\left(x, x^{\prime}\right)$ using plane wave modes. In the Cartesian coordinate system this is given in components as (see, e.g., Ref. [29])

$$
\begin{aligned}
& G_{\text {tata }}^{(C)}\left(x, x^{\prime}\right)=-4 \pi\left\{1+2\left[\left(\zeta_{a}\right)^{2}-\left(\zeta_{t}\right)^{2}\right] \xi^{-2}\right\} G_{6}^{+}\left(x, x^{\prime}\right), \\
& G_{\text {tatb }}^{(C)}\left(x, x^{\prime}\right)=-8 \pi \zeta_{a} \zeta_{b} \xi^{-2} G_{6}^{+}\left(x, x^{\prime}\right), \\
& G_{\text {taab }}^{(C)}\left(x, x^{\prime}\right)=G_{a b t a}^{(C)}\left(x, x^{\prime}\right)=-8 \pi \zeta_{t} \zeta_{b} \xi^{-2} G_{6}^{+}\left(x, x^{\prime}\right), \\
& G_{a b a b}^{(C)}\left(x, x^{\prime}\right)=4 \pi\left\{1+2\left[\left(\zeta_{a}\right)^{2}+\left(\zeta_{b}\right)^{2}\right] \xi^{-2}\right\} G_{6}^{+}\left(x, x^{\prime}\right), \\
& G_{a c b c}^{(C)}\left(x, x^{\prime}\right)=8 \pi \zeta_{a} \zeta_{b} \xi^{-2} G_{6}^{+}\left(x, x^{\prime}\right),
\end{aligned}
$$

where $a, b, c$ take values $x, y, z$ with $a, b$ and $c$ being all distinct (no summation convention is used here) and $\zeta^{\alpha}$ $=x^{\alpha}-x^{\prime \alpha}$. We have defined $G_{6}^{+}\left(x, x^{\prime}\right)=\left(4 \pi^{3} \xi^{4}\right)^{-1}$, where

$$
\begin{aligned}
\xi^{2} & =\left(t-t^{\prime}-i \epsilon\right)^{2}-\left(x-x^{\prime}\right)^{2}-\left(y-y^{\prime}\right)^{2}-\left(z-z^{\prime}\right)^{2} \\
& =\left(t-t^{\prime}-i \epsilon\right)^{2}-r^{2}-r^{\prime 2}+2 r r^{\prime} \cos \gamma .
\end{aligned}
$$

The components $G_{t r t r}$ in spherical polar coordinates can be obtained from the Cartesian ones (A9)-(A13) by using standard tensor transformation: 


$$
\begin{aligned}
G_{t r t r}\left(x, x^{\prime}\right)= & \frac{4 \pi}{r r^{\prime}}\left(\left(x x^{\prime}+y y^{\prime}+z z^{\prime}\right) \xi^{2}\right. \\
& +2\left[\left(x y^{\prime}-x^{\prime} y\right)^{2}+\left(x z^{\prime}-x^{\prime} z\right)^{2}\right. \\
& \left.\left.+\left(y z^{\prime}-y^{\prime} z\right)^{2}\right]\right) \frac{G_{6}^{+}\left(x, x^{\prime}\right)}{\xi^{2}}, \\
= & \frac{1}{\pi^{2} \xi^{6}}\left\{\left[\left(t-t^{\prime}-i \epsilon\right)^{2}-r^{2}-r^{\prime 2}\right]\right. \\
& \left.\times \cos \gamma+2 r r^{\prime}\right\} .
\end{aligned}
$$

Similarly, we find

$$
\begin{aligned}
G_{\theta \phi \theta \phi}\left(x, x^{\prime}\right)= & \frac{r^{2} r^{\prime 2} \sin \theta \sin \theta^{\prime}}{\pi^{2} \xi^{6}} \\
& \times\left\{\left[\left(t-t^{\prime}-i \epsilon\right)^{2}-r^{2}-r^{\prime 2}\right] \cos \gamma+2 r r^{\prime}\right\} .
\end{aligned}
$$

Equations (A15) and (A4) and Eqs. (A16) and (A8) can be shown to agree by using the formula

$$
\sum_{l=1}^{\infty} l(l+1)(2 l+1) P_{l}(t) Q_{l}(z)=\frac{2(t z-1)}{(z-t)^{3}},
$$

which can be proved by applying $(d / d t)\left[\left(t^{2}-1\right) d / d t\right]$ on both sides of the well-known formula

$$
\sum_{l=0}^{\infty}(2 l+1) P_{l}(t) Q_{l}(z)=1 /(z-t)
$$

and using the Legendre equation satisfied by $P_{l}(t)$.

\section{APPENDIX B: A DERIVATION OF EQ. (4.19)}

Define

$$
F(z) \equiv \sum_{l=1}^{\infty} \frac{2 l+1}{l(l+1)}\left[\frac{d}{d z} Q_{l}(z)\right]^{2}
$$

By the Legendre equation satisfied by $Q_{l}(z)$, we have

$$
\begin{aligned}
& \frac{d}{d z}\left\{\left(z^{2}-1\right)^{2}\left[\frac{d}{d z} Q_{l}(z)\right]^{2}\right\} \\
& \quad=2 l(l+1)\left(z^{2}-1\right) Q_{l}(z) \frac{d}{d z} Q_{l}(z) .
\end{aligned}
$$

Hence,

$$
\frac{d}{d z}\left[\left(z^{2}-1\right)^{2} F(z)\right]=\left(z^{2}-1\right) \frac{d}{d z}\left\{\sum_{l=1}^{\infty}(2 l+1)\left[Q_{l}(z)\right]^{2}\right\} .
$$

$$
\sum_{l=0}^{\infty}(2 l+1)\left[Q_{l}(z)\right]^{2}=\frac{1}{z^{2}-1} .
$$

[This can be obtained by squaring both sides of Eq. (A18) and integrating over the variable $t$ from -1 to 1.] By substituting Eq. (B4) in Eq. (B3), we obtain

$$
\begin{aligned}
\frac{d}{d z}\left[\left(z^{2}-1\right)^{2} F(z)\right] & =\left(z^{2}-1\right) \frac{d}{d z}\left[\frac{1}{z^{2}-1}-\frac{1}{4}\left(\ln \frac{z+1}{z-1}\right)^{2}\right] \\
& =-\frac{2 z}{z^{2}-1}+\ln \frac{z+1}{z-1}
\end{aligned}
$$

where we have used

$$
Q_{0}(z)=\frac{1}{2} \ln \frac{z+1}{z-1} .
$$

Integration of Eq. (B5) leads to

$$
\left(z^{2}-1\right)^{2} F(z)=z \ln \frac{z+1}{z-1}-2+C,
$$

where $C$ is a constant. Recalling that $Q_{l}(z) \sim z^{-l-1}$ as $z$ $\rightarrow \infty$, we find that the left-hand side tends to zero in this limit. Therefore $C=0$. Hence,

$$
F(z)=\frac{1}{\left(z^{2}-1\right)^{2}}\left(z \ln \frac{z+1}{z-1}-2\right)=\frac{2 Q_{1}(z)}{\left(z^{2}-1\right)^{2}} .
$$

\section{APPENDIX C: ABSORPTION CROSS SECTION IN SPHERICAL POLAR COORDINATES: A DERIVATION OF EQ. (5.1)}

We consider the divergence-free vector eigenfunctions of the Laplacian on the $(p+1)$-dimensional Euclidean space. That is, we examine the solutions of the following equations:

$$
\nabla^{b}\left(\nabla_{b} B_{a}-\nabla_{a} B_{b}\right)=-\omega^{2} B_{a}
$$

and

$$
\nabla^{a} B_{a}=0
$$

Let $\mathbf{x}=\left(x_{1}, x_{2}, \ldots, x_{p+1}\right)$ be the Cartesian coordinates. There are plane wave solutions of the form

$$
B_{j}^{(C ; \omega \hat{k} \hat{\epsilon})}=\hat{\epsilon}_{j} e^{i \mathbf{k} \cdot \mathbf{x}}
$$

with $\omega>0$, where $\hat{k}$ is a unit vector and $\mathbf{k}=\omega \hat{k}$, and $\hat{\epsilon}$ is a polarization unit vector orthogonal to $\hat{k}$. We define

$$
\left(B^{(1)}, B^{(2)}\right)_{\mathrm{E}} \equiv \int d^{p+1} \overline{\mathbf{x}} \overline{B^{(1)}} B^{(2) a},
$$

for any vectors $B_{a}^{(1)}$ and $B_{a}^{(2)}$ on this space. Then

$$
\left(B^{(C ; \omega \hat{k} \hat{\epsilon})}, B^{\left(C ; \omega^{\prime} \hat{k}^{\prime} \hat{\epsilon}^{\prime}\right)}\right)_{\mathrm{E}}=(2 \pi)^{p+1} \overline{\hat{\epsilon}} \cdot \hat{\boldsymbol{\epsilon}}^{\prime} \delta^{p+1}\left(\mathbf{k}-\mathbf{k}^{\prime}\right) .
$$

Now recall that $[5]$ 
Now, suppose that $B_{a}^{(P ; \omega \kappa)}$ are solutions of Eqs. (C1) and (C2) with a discrete label $\kappa$ satisfying

$$
\left(B^{(P ; \omega \kappa)}, B^{\left(P ; \omega^{\prime} \kappa^{\prime}\right)}\right)_{\mathrm{E}}=2 \pi \delta_{\kappa \kappa^{\prime}} \delta\left(\omega-\omega^{\prime}\right) .
$$

Suppose that the plane wave solutions are expanded as

$$
B_{a}^{(C ; \omega \hat{k} \hat{\epsilon})}=\sum_{\kappa} \alpha(\omega, \hat{k}, \hat{\epsilon} ; \kappa) B_{a}^{(P ; \omega \kappa)},
$$

where $\alpha(\omega, \hat{k}, \hat{\epsilon} ; \kappa)$ are constants. As can be seen from the mode analysis in Sec. II, a solution of Eqs. (C1) and (C2) satisfies the normalization condition (C6) if its incoming angular components at large $r$ are $B_{i} \sim r^{-p / 2} e^{-i \omega r} Y_{i}$, with the spherical harmonic on $p$-sphere $Y_{i}$ being normalized by $\int d \Omega_{p} \bar{Y}^{i} Y_{i}=1$. (The component $B_{r}$ can be shown to be suppressed by an extra power of $r$.) Thus, if we define the flux so that the solution $B_{a}^{(C ; \omega \hat{k} \hat{\epsilon})}$ has a unit flux, then its content of the incoming mode $B_{a}^{(P ; \omega \kappa)}$ is $|\alpha(\omega, \hat{k}, \hat{\epsilon} ; \kappa)|^{2}$ per unit time.

By using orthonormality of these solutions, we find

$$
\alpha(\omega, \hat{k}, \hat{\epsilon} ; \kappa) 2 \pi \delta\left(\omega-\omega^{\prime}\right)=\left(B^{(P ; \omega \kappa)}, B^{\left(C ; \omega^{\prime} \hat{k} \hat{\epsilon}\right)}\right)_{\mathrm{E}} .
$$

From this one readily finds

$$
\begin{aligned}
& \mid \alpha\left.(\omega, \hat{k}, \hat{\epsilon} ; \kappa)\right|^{2}(2 \pi)^{2} \omega^{p} \delta\left(\omega-\omega^{\prime \prime}\right) \\
& \quad=\int_{0}^{\infty} d \omega^{\prime} \omega^{\prime p}\left(B^{(P ; \omega \kappa)}, B^{\left(C ; \omega^{\prime} \hat{k} \hat{\epsilon}\right)}\right) \mathrm{E}\left(B^{\left(C ; \omega^{\prime} \hat{k} \hat{\epsilon}\right)}, B^{\left(P ; \omega^{\prime \prime} \kappa\right)}\right) \\
& \mathrm{E}
\end{aligned}
$$

Now, the average of $\overline{\hat{\epsilon}}_{j} e^{i \omega^{\prime} \hat{k} \cdot \mathbf{x}} \hat{\epsilon}_{l} e^{-i \omega^{\prime} \hat{k} \cdot \mathbf{x}^{\prime}}$ over the polarization vectors is

$$
\frac{1}{p}\left(\delta_{j l}-\hat{k}_{j} \hat{k}_{l}\right) e^{i \omega^{\prime} \hat{k} \cdot\left(\mathbf{x}-\mathbf{x}^{\prime}\right)}=\frac{1}{p}\left(\delta_{j l}-\frac{1}{\omega^{2}} \partial_{j} \partial_{l}^{\prime}\right) e^{i \omega^{\prime} \hat{k} \cdot\left(\mathbf{x}-\mathbf{x}^{\prime}\right)},
$$

where the partial derivative $\partial_{j}$ is with respect to $\mathbf{x}$ whereas the partial derivative $\partial_{l}^{\prime}$ is with respect to $\mathbf{x}^{\prime}$. Since the modes $B_{a}^{\left(P^{\prime} \omega \kappa\right)}$ are divergence-free, these derivatives can be dropped when we average Eq. (C9) over the polarization vectors. By averaging Eq. (C9) over $\hat{\epsilon}$ and $\hat{k}$ and then using

$$
\int d \omega^{\prime} \omega^{\prime p} d \hat{k} e^{i \omega^{\prime} \hat{k} \cdot\left(\mathbf{x}-\mathbf{x}^{\prime}\right)}=(2 \pi)^{p} \delta^{p+1}\left(\mathbf{x}-\mathbf{x}^{\prime}\right),
$$

we find $|\alpha(\omega ; \kappa)|^{2}$, the average of $|\alpha(\omega, \hat{k}, \hat{\epsilon} ; \kappa)|^{2}$ over $\hat{k}$ and $\hat{\epsilon}$, as

$$
|\alpha(\omega ; \kappa)|^{2} \omega^{p} \delta\left(\omega-\omega^{\prime \prime}\right)=\frac{(2 \pi)^{p-1}}{p \Omega_{p}}\left(B^{(P ; \omega \kappa)}, B^{\left(P, \omega^{\prime \prime} \kappa\right)}\right)_{\mathrm{E}} .
$$

Then from Eq. (C6), we find

$$
|\alpha(\omega ; \kappa)|^{2}=\frac{(2 \pi)^{p}}{p \Omega_{p} \omega^{p}} .
$$

Thus, if a plane wave has a unit flux, it contains the incoming mode with label $\kappa$ given by Eq. (C12) per unit time on average.

The angular components $A_{i}$ of physical modes I are gradients of scalar spherical harmonics. On the other hand, physical modes II are divergence-free vectors on the $p$-sphere. Let $M_{l}^{(1)}$ and $M_{l}^{(2)}$ be the multiplicities of these two types of modes with a given angular momentum $l$. Then the number of physical modes I with angular momentum $l$ is $M_{l}^{(1)}$ and that of physical modes II is $M_{l}^{(2)}$. Then, if there is a plane wave with a unit flux, the content of physical modes I and II with angular momentum $l$ are $\left[(2 \pi)^{p} / p \Omega_{p} \omega^{p}\right] M_{l}^{(1)}$ and $\left[(2 \pi)^{p} / p \Omega_{p} \omega^{p}\right] M_{l}^{(2)}$ per unit time, respectively. Therefore, if the absorption probabilities of physical modes I and II by a spherical black hole are $\mathcal{P}_{l}^{(1)}$ and $\mathcal{P}_{l}^{(2)}$, respectively, then the absorption cross section is given by Eq. (5.1).
[1] S. W. Hawking, Commun. Math. Phys. 43, 199 (1975).

[2] P. Candelas and D. W. Sciama, Phys. Rev. D 27, 1715 (1983).

[3] B. P. Jensen and P. Candelas, Phys. Rev. D 33, 1590 (1986); 35, 4041(E) (1987).

[4] P. Candelas, Phys. Rev. D 21, 2185 (1980).

[5] A. Higuchi, G. E. A. Matsas, and D. Sudarsky, Phys. Rev. D 56, R6071 (1997); 58, 104021 (1998).

[6] L. C. B. Crispino, A. Higuchi, and G. E. A. Matsas, Class. Quantum Grav. 17, 19 (2000).

[7] L. C. B. Crispino, A. Higuchi, and G. E. A. Matsas, Phys. Rev. D 58, 084027 (1998).

[8] G. Cognola and P. Lecca, Phys. Rev. D 57, 1108 (1998).

[9] W. G. Unruh, Phys. Rev. D 14, 870 (1976).

[10] S. S. Gubser, Phys. Rev. D 56, 7854 (1997).

[11] S. A. Teukolsky, Phys. Rev. Lett. 29, 1114 (1972); Astrophys. J. 185, 635 (1973).
[12] A. A. Starobinskii and S. M. Churilov, Zh. Éksp. Teor. Fiz. 65, 3 (1973) [Sov. Phys. JETP 38, 1 (1974)].

[13] E. T. Newman and R. Penrose, J. Math. Phys. 3, 566 (1962); 4, 998(E) (1963).

[14] C. Itzykson and J.-B. Zuber, Quantum Field Theory (McGrawHill, New York, 1980).

[15] A. Chodos and E. Myers, Ann. Phys. (N.Y.) 156, 412 (1984).

[16] R. Camporesi and A. Higuchi, J. Geom. Phys. 15, 57 (1994).

[17] J. L. Friedman, Commun. Math. Phys. 62, 247 (1978).

[18] R. C. Myers and J. Perry, Ann. Phys. (N.Y.) 172, 304 (1986).

[19] R. M. Wald, Quantum Field Theory in Curved Spacetime and Black Hole Thermodynamics, Chicago Lectures in Physics (University of Chicago, Chicago, 1994).

[20] D. G. Boulware, Phys. Rev. D 11, 1404 (1975); 12, 350 (1975).

[21] J. B. Hartle and S. W. Hawking, Phys. Rev. D 13, 2188 (1976). 
[22] B. S. Kay and R. M. Wald, Phys. Rep. 207, 49 (1991).

[23] T. Kugo and I. Ojima, Phys. Lett. 73B, 459 (1978); Suppl. Prog. Theor. Phys. 66, 1 (1979).

[24] A. Higuchi, G. E. A. Matsas, and D. Sudarsky, Phys. Rev. D 46, 3450 (1992).

[25] I. S. Gradshteyn and I. M. Ryzhik, Table of Integrals, Series and Products (Academic, New York, 1980); M. Abramowitz and I. A. Stegun, Handbook of Mathematical Functions (Do- ver, New York, 1965).

[26] J. Castiñeiras and G. E. A. Matsas, Phys. Rev. D 62, 064001 (2000).

[27] S. R. Das, G. Gibbons, and S. D. Mathur, Phys. Rev. Lett. 78, 417 (1997).

[28] T. Regge and J. Wheeler, Phys. Rev. 108, 1063 (1957).

[29] S. Takagi, Prog. Theor. Phys. 88, 1 (1986). 